\title{
Effect of Heat Treatment on Band Gap of $\mathrm{V}_{2} \mathrm{O}_{5}$
}

\author{
Rashi Nathawat ${ }^{1, *}$, Ashish K. Kumawat ${ }^{1}$, Satyapal S. Rathore ${ }^{1}$, Anoop K. Mukhopadhyay ${ }^{2}$, K. Kabra ${ }^{3}$ \\ ${ }^{1}$ Functional Ceramics \& Smart Materials Lab, Department of Physics, Manipal University Jaipur, \\ Jaipur 303007, India \\ 2 Department of Physics, Manipal University Jaipur, Jaipur-303007, India \\ ${ }^{3}$ Department of Physics, University of Kota, Rajasthan, India
}

(Received 09 January 2021; revised manuscript received 12 February 2021; published online 25 February 2021)

\begin{abstract}
The most stable oxide of the vanadium oxide family is $\mathrm{V}_{2} \mathrm{O}_{5}$. A lot of research effort is focused on it because it has a multitude of functional applications. Here we report on how the heat treatment $\left(600^{\circ} \mathrm{C}, 5 \mathrm{~h}\right.$, air) affects the microstructure and hence, the band gap of $\mathrm{V}_{2} \mathrm{O}_{5}$. The $\mathrm{V}_{2} \mathrm{O}_{5}$ powders, initially obtained by simple thermal dissociation $\left(500{ }^{\circ} \mathrm{C}, 3 \mathrm{~h}\right.$, air) of ammonium metavanadate, followed by heat treatment of pellets; were studied. The structural and optical studies performed using X-ray diffraction (XRD), FESEM and UV-Vis techniques, provide uniquely interesting results which indicate the possibility of band gap tuning by controlling the microstructure.
\end{abstract}

Keywords: Vanadium Oxide, Multifunctional, Band gap tuning, Microstructure, Heat treatment.

DOI: 10.21272/jnep.13(1).01030

\section{INTRODUCTION}

In the periodic table Vanadium is a $d$-transition metal element with various oxidation states. Therefore, vanadium oxides (V-O) exist in nature in various stereochemistry such as $\mathrm{VO}_{2}, \mathrm{~V}_{2} \mathrm{O}_{5}, \mathrm{~V}_{2} \mathrm{O}_{3}, \mathrm{~V}_{3} \mathrm{O}_{5}, \mathrm{~V}_{4} \mathrm{O}_{7}$, and $\mathrm{V}_{6} \mathrm{O}_{13}[1]$.

Among these the vanadium pentoxide $\left(\mathrm{V}_{2} \mathrm{O}_{5}\right)$ is the most stable and widely studied material in the $\mathrm{V}-\mathrm{O}$ family. The electronic structure of vanadium ions in $\mathrm{V}_{2} \mathrm{O}_{5}$ has $\mathrm{V}^{5+}$ coordination. In such type of transition metal oxides, the $d$-electrons are spatially confined in partially filled orbitals. They strongly interact with each other, because of the prevalent Coulombic repulsion. These materials are extremely sensitive to small changes in external stimuli such as temperature, pressure, and doping [2, 3]. Vanadium oxides are oxyanions of the transition metal vanadium. It shows semiconductor to metal transition (SMT) or insulator to metal transition (IMT). The outstanding properties of $\mathrm{V}_{2} \mathrm{O}_{5}$ such as band gap in visible region (2.2-2.7 eV), worthy chemical and thermal stability, excellent thermoelectric properties make it suitable for various applications such as solar cell, gas sensors, light modulators, lithium-ion batteries, optical-electrical switches, electrochromic devices, optoelectronic devices, and infrared windows [4-9]. These inorganic materials exhibit highly photochromic and anisotropic electrical properties [6]. Due to its response in visible radiation range $\mathrm{V}_{2} \mathrm{O}_{5}$ is used as a high-performance photo catalyst which absorbs photon and speed up the chemical reaction [10]. The multi-valance layered structure of $\mathrm{V}_{2} \mathrm{O}_{5}$ demonstrate high thermo-chromic and electrochemical properties for wide optical band gap. As the most stable among other vanadium oxides, $\mathrm{V}_{2} \mathrm{O}_{5}$ can be synthesized by various techniques such as chemical different methods, the so-called thermal dissociation is the relatively simple, cost effective, environment friendly vapor deposition, magnetron sputtering, sol-gel method, pulsed

* rashi.nathawat@gmail.com

The results were presented at the International Conference on Multifunctional Nanomaterials (ICMN2020) laser deposition, electron beam evaporation, electrospinning, spray pyrolysis, hydrothermal and solid-state sintering [11-16]. Amongst and fast technique to prepare micron to sub-micron size powders of $\mathrm{V}_{2} \mathrm{O}_{5}$ in short reaction time.In the present study, single phase $\mathrm{V}_{2} \mathrm{O}_{5}$ has been synthesized by thermal dissociation method. Next these powders were consolidated, and heat treated to induce densification by possible solid-state sinteringBoth types of samples were characterized by XRD, FESEM and UV-Vis spectroscopy techniques.

\section{EXPERIMENTAL DETAILS}

To prepare the $\mathrm{V}_{2} \mathrm{O}_{5}$ powders Ammonium metavanadate $\left(\mathrm{NH}_{4} \mathrm{VO}_{3}, 99.0 \%\right.$, ACS reagent, Sigma Aldrich) was used without any further purification. In the thermal dissociation process, requisite mass of $\mathrm{NH}_{4} \mathrm{VO}_{3}$ powder was heat treated at $500{ }^{\circ} \mathrm{C}$ in a porcelain crucible for $3 \mathrm{~h}$ in air. It resulted in an orange colored $\mathrm{V}_{2} \mathrm{O}_{5}$ powder due to the following reaction [1]:

Using these $\mathrm{V}_{2} \mathrm{O}_{5}$ powders, the orange pellets $(\sim 1 \mathrm{~mm}$ thick and $\sim 10 \mathrm{~mm}$ diameter) were prepared using a uniaxial press. The pellets were subsequently heat treated at $600{ }^{\circ} \mathrm{C}$ for $5 \mathrm{~h}$ in air to induce densification by possible solid-state sintering. The as prepared powders and heat treated (HT) pellets were utilized for further studies. The structural characterizations were performed by XRD (Rigaku, Ultima-IV) technique using $\mathrm{Cu} \mathrm{K}_{\alpha}(\lambda=0.154 \mathrm{~nm})$ monochromatic radiation source. The XRD scan were recorded for $2 \theta$ range of $10^{\circ}$ to $90^{\circ}$. The surface microstructures were studied by using the field emission scanning electron microscopy (FESEM) technique by utilizing a conventional machine (JEOL, JSM-7610FPlus). The optical properties were studied from 300 to $800 \mathrm{~nm}$ by using a standard UV-Vis spectrophotometer (Shimadzu, UV-2600).

$$
2 \mathrm{NH}_{4} \mathrm{VO}_{3} \stackrel{\Delta}{\longrightarrow} \mathrm{V}_{2} \mathrm{O}_{5}+2 \mathrm{NH}_{3}+\mathrm{H}_{2} \mathrm{O}
$$




\section{RESULT AND DISCUSSION}

\subsection{X-ray Diffraction}

Figure 1 shows the XRD patterns of as prepared powders and heat-treated $(\mathrm{HT}) \mathrm{V}_{2} \mathrm{O}_{5}$ pellets. The patterns match with that of the standard (ICSD collection code: 060767). Thereby, the present results confirm the formation of single phase $\mathrm{V}_{2} \mathrm{O}_{5}$. It also ensures that both the samples are orthorhombic with lattice parameters of $a=11.51 \AA, b=3.56 \AA$, and $c=4.36 \AA$. The major peaks correspond to the presence of $(h k l)$ e.g., (200), (001), (101), (110), (301), (011), and (310) planes at around $2 \theta$ values of $15.38^{\circ}, 20.31^{\circ}, 21.74^{\circ}, 26.15^{\circ}$, $31.04^{\circ}, 32.39^{\circ}$, and $34.31^{\circ}$, respectively. The corresponding inter-planar spacing (d) values are given by $5.75 \AA, 4.36 \AA, 4.08 \AA, 3.40 \AA, 2.87 \AA, 2.76 \AA$, and $2.61 \AA$, respectively. The major peaks of HT sample are more intense compared to those of the as prepared sample. This enhanced intensity represents more crystalline nature of the powder after the heat treatment. The crystallite size (DDs) was calculated by following Debye-Scherrer (D-S) formula [17]:

$$
D_{D S}=k \lambda / \beta \cos \theta,
$$

where, $k$ is a constant of value of $\sim 0.9, \lambda$ is wavelength of monochromatic source and $\beta$ is the full width at half maximum. Further, $\theta$ is the angle of diffraction. The crystallite sizes of the different planes are mentioned in Table 1 along with their respective miller indices. The average crystallite sizes of both the samples are estimated to be $\sim 23 \mathrm{~nm}$. The crystallite sizes along with the strains were also determined by the well-known Williamson and Hall (W-H) method [18]. The crystallite size $\left(D_{\mathrm{WH}}\right)$ and strain $(\varepsilon)$ are thus estimated from the corresponding $\mathrm{W}-\mathrm{H}$ plots by utilizing the following relation:

$$
\beta \cos \theta=4 \varepsilon \sin \theta+k \lambda / D_{W H}
$$

The relationships between the terms $\beta \cos \theta$ and $4 \sin \theta$ are shown in Figure 2(a) and (b) for the as prepared powders and the HT samples, respectively. The linear fitting shown in these plots estimate the tensile strains of about $0.36 \times 10^{-4}$ and $18.5 \times 10^{-4}$ respectively for the as-prepared and the HT samples. The tensile strain is nearly two orders of magnitude higher in the HT samples reflecting densification-induced strain generation in the microstructure. The higher amount of strain also ensures better intensities of the crystalline peaks in the HT sample. However, the crystallite size for both the samples is still about $23 \mathrm{~nm}$. It supports the results already derived by the D-S technique, as mentioned above. It also confirms that there is no significant change in the fundamental crystalline size after the heat treatment.

\subsection{Microstructural Studies}

The typical FESEM based photomicrographs of the as prepared powders and the HT pellets are shown in Figure 3(a) and (b), respectively. Both the samples have some relatively smaller approximately equiaxed grains along with some rod-shaped grains. These rodshaped grains occasionally reflect resemblance to a hexagonal growth pattern. A very approximate and rough grain size distribution data collection was attempted. These data are shown as histograms placed as insets in Figure 3(a) and Figure 3(b) for the as prepared powders and the HT pellets, respectively. The histograms suggest that average size of grains is about $\sim 440 \mathrm{~nm}$ in the case of the as prepared powders but it almost doubles to $\sim 840 \mathrm{~nm}$ in the case of the HT samples. Heat treatment induced enhancement in grain size is also reported by other researchers although the experimental conditions are not exactly similar [19-20].

\subsection{Optical Properties}

The $\mathrm{V}_{2} \mathrm{O}_{5}$ is a chromogenic material. Its optical properties change on exposure to external stimuli. For instance, incidence of photons causes photochromic changes. Change in applied temperature causes thermochromic changes. Similarly, change in applied voltage causes electrochromic changes. As mentioned earlier, the optical properties of the $\mathrm{V}_{2} \mathrm{O}_{5}$ phase have been studied over visible and near infrared wavelength ranges. The absorbance spectra are shown in Figure 4(a). It exhibits, that the first absorption edge for the HT samples is at around $550 \mathrm{~nm}$ in visible range. It is also present in the as prepared sample, but the absorbance of as prepared sample is relatively lower than that of the HT sample, as expected. The relatively higher crystalline peak-intensities in the XRD pattern of the HT sample explains its relatively higher absorbance. This edge shifts to much lower wavelength of $350 \mathrm{~nm}$ after heat treatment. This occurs due to inter-band transition [21].

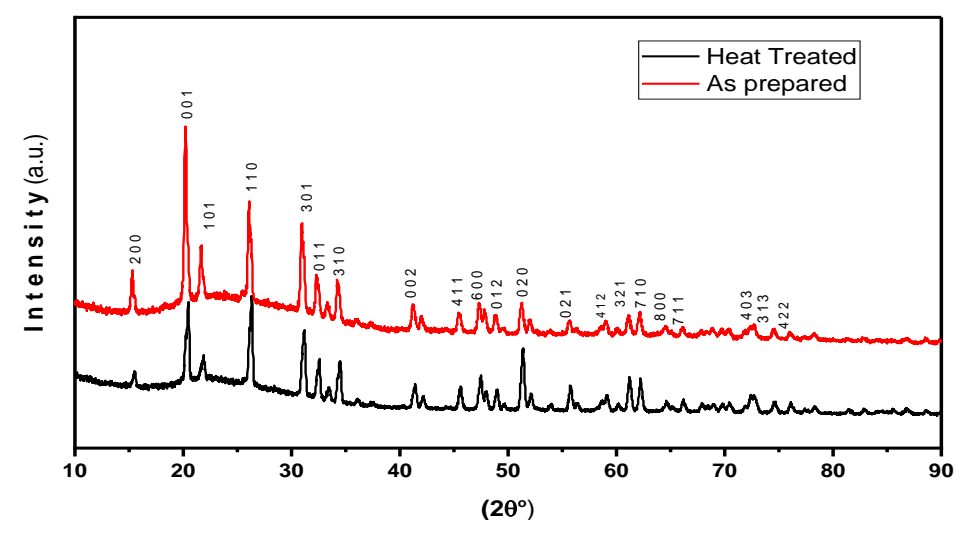

Fig. 1 - XRD pattern of as-prepared and heat-treated $\mathrm{V}_{2} \mathrm{O}_{5}$ 
Table 1: Crystallite size of the material with respective miller indices

\begin{tabular}{|c|c|c|c|c|c|c|c|}
\hline Miller indices $(h k l)$ & $(200)$ & $(001)$ & $(\mathbf{1 0 1 )}$ & $(110)$ & $(301)$ & $(011)$ & (310) \\
\hline \multirow{4}{*}{ FWHM } & \multicolumn{7}{|c|}{ As prepared } \\
\hline & 0.310 & 0.385 & 0.378 & 0.337 & 0.360 & 0.348 & 0.359 \\
\hline & \multicolumn{7}{|c|}{ Heat treated } \\
\hline & 0.315 & 0.300 & 0.335 & 0.353 & 0.378 & 0.388 & 0.375 \\
\hline \multirow{4}{*}{$D_{D S}$} & \multicolumn{7}{|c|}{ As prepared } \\
\hline & $26 \mathrm{~nm}$ & $21 \mathrm{~nm}$ & $21 \mathrm{~nm}$ & $24 \mathrm{~nm}$ & $23 \mathrm{~nm}$ & $24 \mathrm{~nm}$ & $23 \mathrm{~nm}$ \\
\hline & \multicolumn{7}{|c|}{ Heat treated } \\
\hline & $25 \mathrm{~nm}$ & $26 \mathrm{~nm}$ & $24 \mathrm{~nm}$ & $23 \mathrm{~nm}$ & $22 \mathrm{~nm}$ & $21 \mathrm{~nm}$ & $22 \mathrm{~nm}$ \\
\hline
\end{tabular}

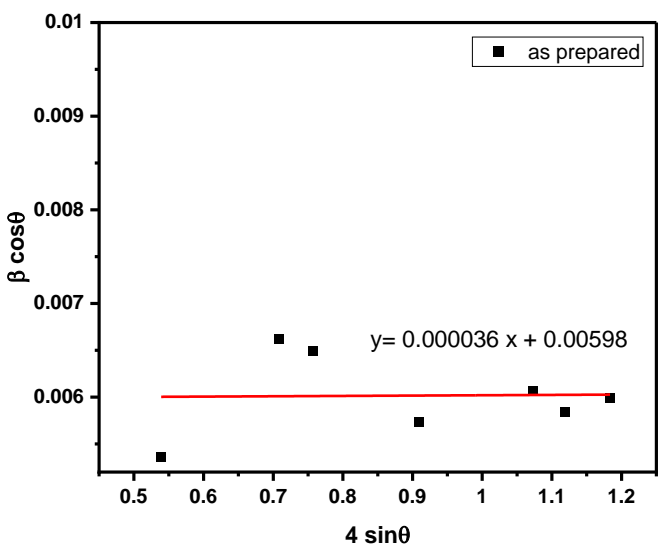

(a)

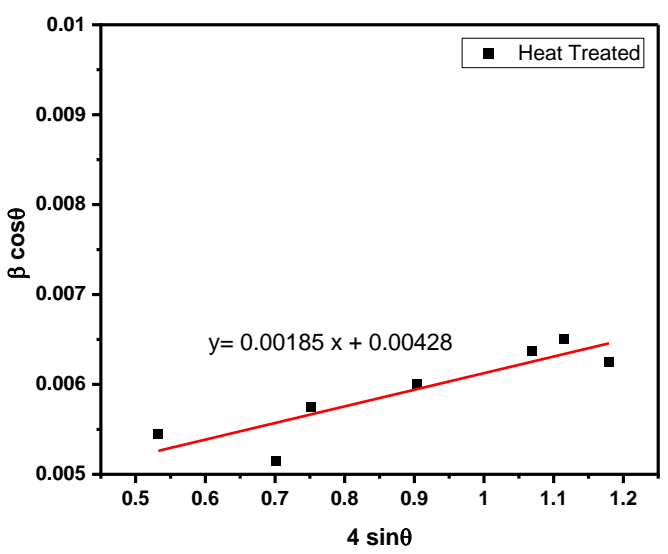

(b)

Fig. 2 - The W-H plots with linear fitted data of (a) as prepared sample (b) heat treated sample

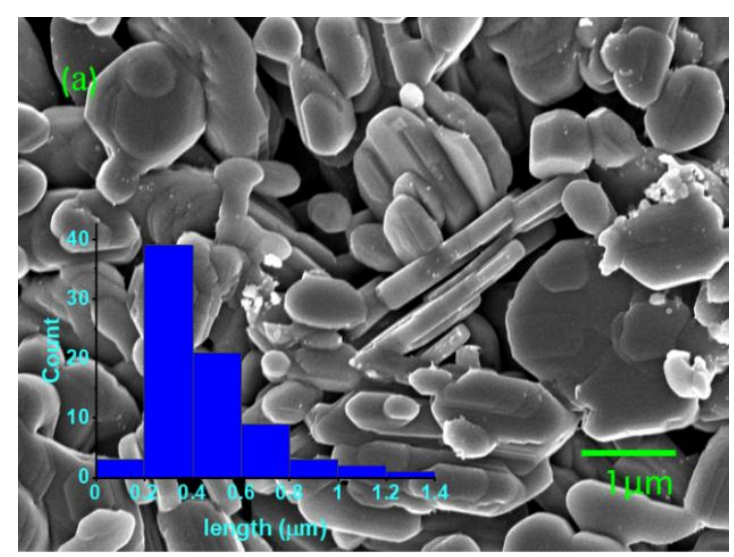

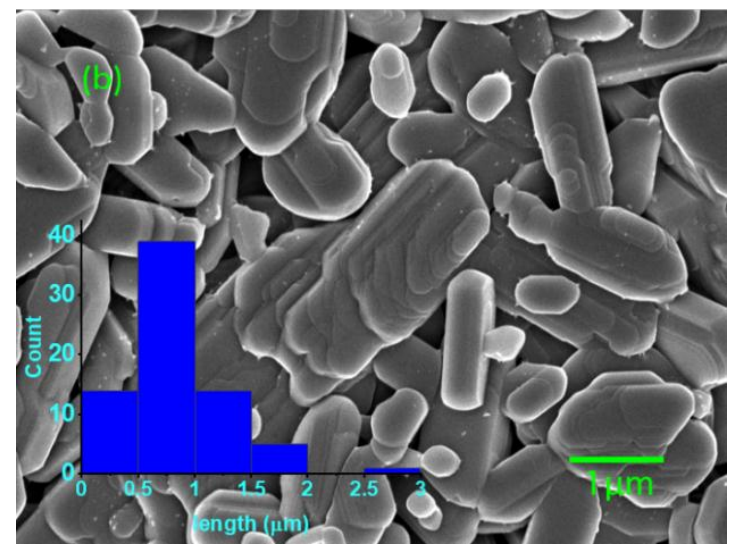

Fig. 3- FESEM image of (a) as prepared $\mathrm{V}_{2} \mathrm{O}_{5}$ and (b) heat treated $\mathrm{V}_{2} \mathrm{O}_{5}$ with histogram of grain size distribution

Figure 4(b) represents the transmittance spectra of the as prepared and HT samples. Since the absorbance increases (Figure 4a) the transmittance decreases after heat treatment, as expected. Thus, the larger grain size of the HT sample reduces transmission. On the other hand, the relatively finer grain size, and a relatively less crystalline microstructure (Figure 3a) of the as prepared sample provides possibly more means of transmission (Fig. 4b).

Figure 4(c) represents the Tauc plots of the as prepared and HT samples. These plots are used to determine the respective optical band gaps of these two samples. The band gaps are calculated by the following relation [22].

$$
\alpha h v=K\left(h v-E_{g}\right)^{n}
$$

where $\alpha$ is absorption coefficient and is calculated by the Beer Lambert law [22]

$$
\begin{gathered}
I / I_{0}=e^{-\alpha_{l}} \\
\alpha=2.303 \times A / L,
\end{gathered}
$$

where, $A$ and $L$ are absorbance and length of the sample holder, respectively. Further, $(h v)$ is incident photon energy, $K$ is energy independent constant and $E_{g}$ is the optical band gap.

For direct band gap transition $n$ equals $1 / 2$ and for indirect band gap transition $n$ equals 2 . Based on the usage of the aforesaid equation and linear intercept on the energy axis, the energy required for indirect band gap transition is estimated to be $\Sigma 2.64 \mathrm{eV}$ for the as prepared sample which is expected for the semiconducting orthorhombic structure for the $\mathrm{V}_{2} \mathrm{O}_{5}$ materials [23]. 
Surprisingly, it reduces to $2.45 \mathrm{eV}$ for the HT sample.

This decrease in band gap signifies that relatively lesser amount of energy is required for indirect band gap transition to occur in the HT sample. The lower is the energy requirement, the higher is the chance of carrier transition across the band gap. The higher is the carrier transition, the larger is the carrier concentration. The higher is the carrier concentration the higher is the conductivity. The higher is the conductivity the lower is the resistivity of the sample. It therefore suggests a road map for semiconductor to metal transition.

Other researchers also reported [24] that the absorption edge in $\mathrm{V}_{2} \mathrm{O}_{5}$ has an Urbach-rule dependence and above $2.4 \mathrm{eV}$ the absorption happens only due to transition at $k \neq 0$. Thus, the present results imply that by tuning the microstructure through heat treatment; a situation like semiconductor state to metallic state transition could be affected in $\mathrm{V}_{2} \mathrm{O}_{5}$ material by a simple, inexpensive approach such as the one used in the current work.
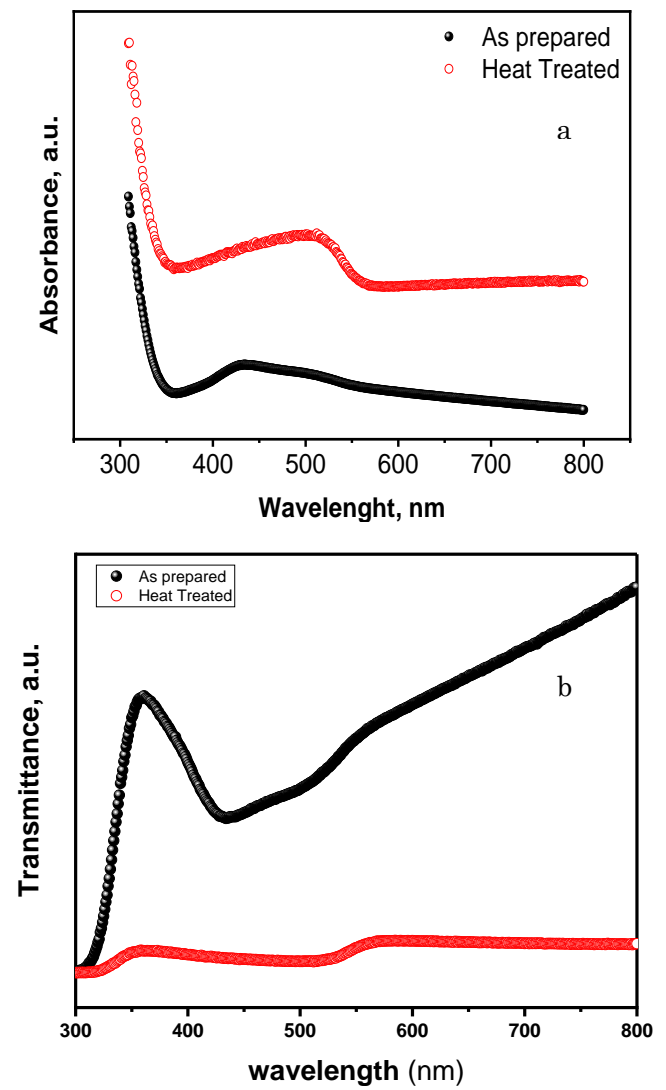

\section{REFERENCES}

1. A.A. Akandeab, B.W. Linganisoa, E.C. Dhongea, B.P. Rammutla, K.E. Machatinec, A. Prinslooc, L. Kunertc, H. Mwakikunga, Mater. Chem. Phys. 151, 206 (2015).

2. M. Kang, S.W. Kim, Y. Hwang, Y. Um, J.W. Ryu, AIP Adv. 3, 052129 (2013)

3. B. Hu, C. Xu, M.K. Aslam, Y. Cen, J. Hu, Y. Li, Y. Liu,

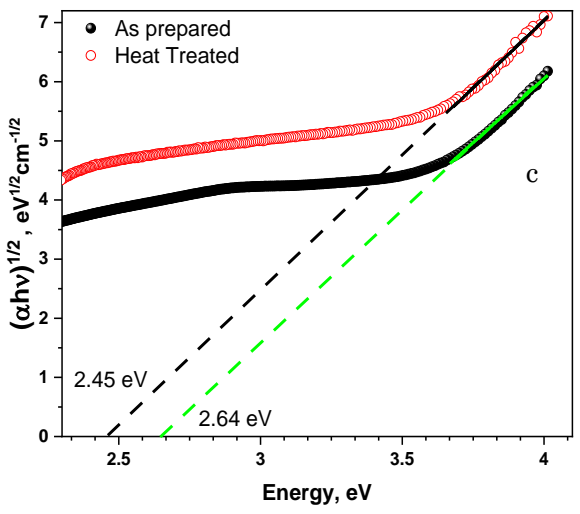

Fig. 4 - (a) Absorbance, (b) Transmission and (c) Tauc plots of as-prepared and heat treated $\mathrm{V}_{2} \mathrm{O}_{5}$

\section{CONCLUSION}

The major achievement of the present study lies in identification of a simple inexpensive technique like heat treatment in air to affect reduction of band gap in $\mathrm{V}_{2} \mathrm{O}_{5}$ material. Thereby it raises a chance to achieve the semiconducting state to metallic state transition as a distinct possibility through tuning of microstructure as well as strain engineering. A simple thermal dissociation results in powders with nanocrystalline crystallite size of about $23 \mathrm{~nm}$ which remains unchanged as pellets from this powder are subsequently densified by heat treatment in air at $600{ }^{\circ} \mathrm{C}$ for $5 \mathrm{~h}$. Both structures are confirmed to have phase pure orthorhombic structure albeit that the grain size almost doubles to about $840 \mathrm{~nm}$ after heat treatment. The stain increases by two orders of magnitude which possibly enhances absorbance but reduces transmission after heat treatment. These factors culminate in achievement of reduction in indirect band gap from $2.64 \mathrm{eV}$ in the as prepared sample to $2.45 \mathrm{eV}$ in the $\mathrm{HT}$ sample, thereby raising a possibility to have tunable band-gap in the current $\mathrm{V}_{2} \mathrm{O}_{5}$ materials.

\section{ACKNOWLEDGEMENTS}

The Authors would like to express special thanks to Sophisticated Analytical Instrument Facility (SAIF) and Central Analytical Facilities (CAF,) Manipal University Jaipur, for the provisions of FESEM and UVVIS facilities; respectively for characterization. One of the author (RN) would like to acknowledge Dr. Ghanshyam Sharma, University of Kota for providing XRD facilities. Further, the author $(\mathrm{RN})$ also acknowledges the award of the Enhanced Seed Grant Project No. (EF/2019-21/QE04-03) to her by Endowment Fund Committee under Directorate of Research at Manipal University Jaipur.
C. Guo, D. Yu, C. Chen, Chem. Eng. J. 389, 123534 (2020).

4. Z.N. Kayani, H. Bashir, S. Riaz, S. Naseem, Optical Properties and Antibacterial Activity of $V$ Doped $\mathrm{ZnO}$ Used in Solar Cells and Biomedical Applications (Elsevier Ltd: 2019).

5. G. Eranna, B.C. Joshi, D.P. Runthala, R.P. Gupta, Critical Rev. Solid State Mater. Sci. 29, 111 (2004). 
6. F.A. Sabah, I.A. Razak, E.A. Kabaa, M.F. Zaini, A.F. Omar, Opt. Mater. 107, 110117 (2020).

7. C. Zhu, J. Shu, X. Wu, P. Li, X. Li, J. Electroanal. Chem. 759, 184 (2015).

8. Z. Wan, H. Mohammad, Y. Zhao, R.B. Darling, M.P. Anantram, IEEE Electron Dev. Lett. 39, 1290 (2018).

9. B. Li, J. Yao, S. Tian, Z. Fang, S. Wu, B. Liu, X. Gong, H. Tao, X. Zhao, Ceram. Int. 46, 18274 (2020).

10. A.T. Raj, K. Ramanujan, S. Thangavel, S. Gopalakrishan, N. Raghavan, G. Venugopal, J. Nanosci. Nanotechnol. 15, 3802 (2015).

11. J. Livage, Materials 3, 4175 (2010).

12. X. Liu, J. Zeng, H. Yang, K. Zhou, D. Pan, RSC Adv. 8, 4014 (2018).

13. M. Farahmandjou, J. Nanomed. Res. 5 No 1, 00103 (2017).

14. M. Al Zoubi, H.K. Farag, F. Endres, J. Mater. Sci. 44, 1363 (2009).

15. C. Paper, A. Akande, S. Africa, A. Akande, 3rd International Conference NSNOCON 014 (Pune: India: 2014).

16. E. Kianfar, Microchem. J. 145, 966 (2019).
17. B. Bera, P. Sekhar Das, M. Bhattacharya, S. Ghosh, A.K. Mukhopadhyay, A. Dey, J. Phys. D: Appl. Phys. 49, 85303 (2016).

18. N. Singh, A. Umar, N. Singh, H. Fouad, O.Y. Alothman, F.Z. Haque, Mater. Res. Bull. 108, 266 (2018).

19. F. Nagashima, Y. Nakagawa, M. Yoshino, Procedia Manufac. 50, 248 (2020).

20. M.A. Hasan, A. Dey, A.C.M. Esther, P. Maiti, A.K. Mukhopadhyay, A. Rajendra, Bull. Mater. Sci. 43, $287(2020)$

21. K. Schneider, J. Mater. Sci.: Mater. Electron. 31, 10478 (2020).

22. C.V. Ramana, O.M. Hussein, Adv. Mater. Opt. Electron. 7, 225 (1997)

23. R. Thangarasu, E. Thangavel, J. Chandrasekaran, O.N. Balasundaram, J. Mater. Sci.: Mater. Electron. 30, 4238 (2019).

24. M. Subhadra, S. Sulochana, P. Kistaiah, Mater. Today: Proc. 5, 26417 (2018) 